\title{
Motor Vehicle Crash (MVC) Case Definitions and How They Impact MVC Surveillance
}

\author{
Jennifer L. Jones*, Dennis M. Falls, Clifton A. Barnett, Amy Ising and Anna E. Waller \\ University of North Carolina at Chapel Hill, Chapel Hill, NC, USA
}

\section{Objective}

Identify and describe how the case definition used to identify MVC patients can impact results when conducting MVC surveillance using ED data. We compare MVC patients identified using external cause of injury codes (E-codes), text searches of triage notes and chief complaint, or both criteria together.

\section{Introduction}

In 2012, an estimated 2.5 million people presented to the ED for a MVC injury in the U.S. ${ }^{1}$ National injury surveillance is commonly captured using E-codes. ${ }^{2}$ However, use of E-codes alone to capture MVC-related ED visits may result in a different picture of MVC injuries compared to using text searches of triage or chief compliant notes.

\section{Methods}

MVC-related ED visit data were obtained from the North Carolina Disease Event Tracking and Epidemiologic Collection Tool (NC DETECT) from one central county in NC for the year 2013. Data were categorized based on case definition used to identify the record as pertaining to an MVC. The three case definitions were: 1) MVC injuries identified using E-codes (E810-E825), 2) text searches for MVC-related key words in either the triage note or chief complaint field, and 3) MVC injuries identified using both text searches and E-codes. Demographic and descriptive characteristics included: sex, age, disposition, transport mode, payor source, visit time, and injury diagnosis (based on the Barell Injury Matrix ${ }^{3}$ ). Descriptive statistics were used to describe differences in patient characteristics based on the case definition used to identify MVC injury. Analyses were conducted using SAS Version 9.2 (Cary, NC) and Microsoft Excel 2007.

\section{Results}

Most ED visits contained both MVC-text and MVC-related E-codes $(n=13422,76 \%)$. Another 4265 visits were identified by including the additional case definitions of text only $(n=2139,12 \%)$ or E-code only ( $\mathrm{n}=2101,12 \%)$. Patients identified using E-codes only were more likely to be male, arrive by ambulance, and admitted to the hospital compared to patients identified by text searches or both text and E-codes. Review of triage notes for those patients without E-codes suggests that patients identified with text searches are more likely to be presenting to the ED for late effects or chronic injuries from MVCs in the past.

\section{Conclusions}

The choice of case definition used for MVC surveillance appears to impact the picture of MVC injury severity. When developing a research question or surveillance project, it is important that public health researchers are aware of the impact case definition has on their results.

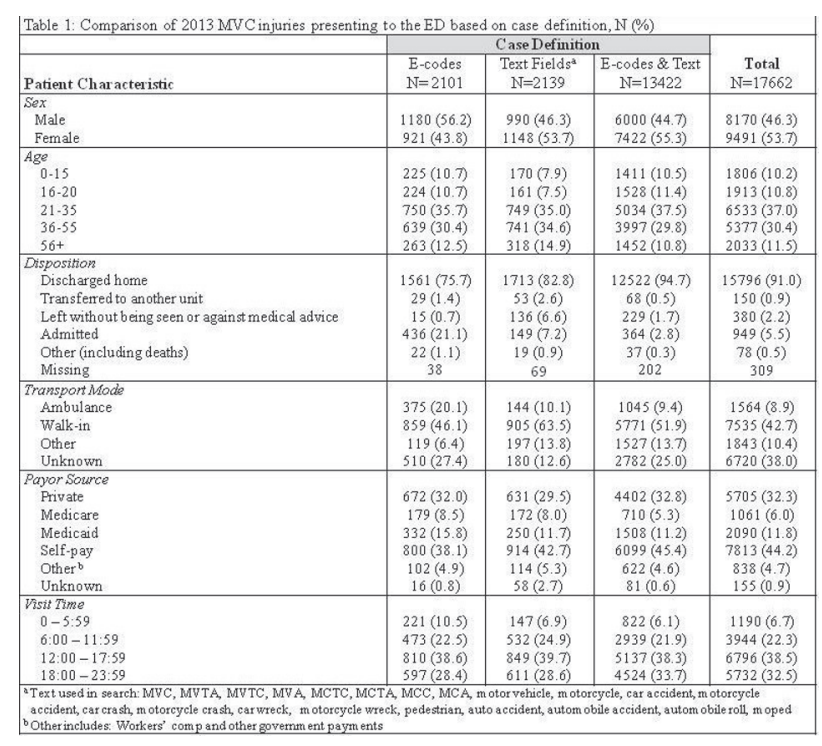

Barell Injury Diagnoses Among MVC-related ED Visits

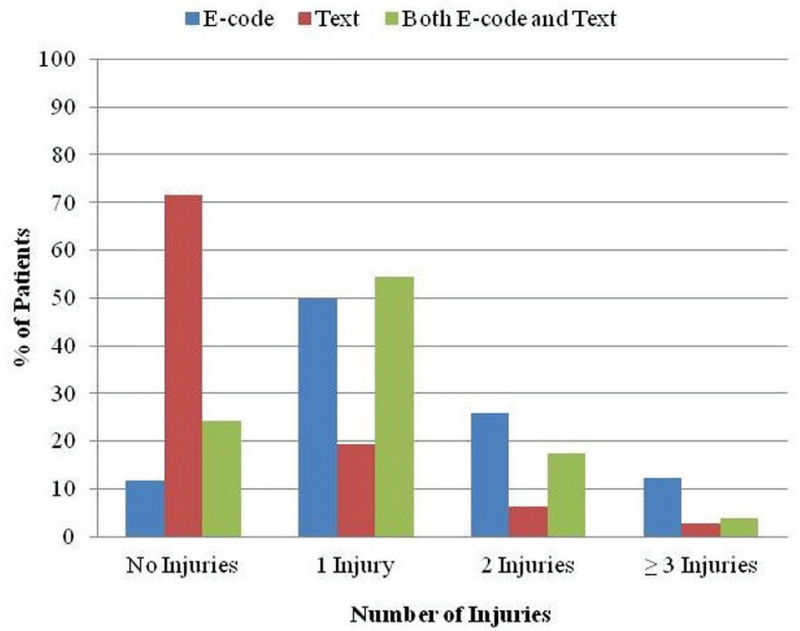

Keywords

MVC Injury; surveillance; ED visit data 


\section{ISDS 2015 Conference Abstracts}

\section{Acknowledgments}

NC DETECT is funded with federal funds by North Carolina Division of Public Health (NC DPH), Public Health Emergency Preparedness Grant (PHEP), and managed through a collaboration between NC DPH and the UNC Department of Emergency Medicine's Carolina Center for Health Informatics (UNC CCHI). The NC DETECT Data Oversight Committee does not take responsibility for the scientific validity or accuracy of methodology, results, statistical analyses, or conclusions presented. The NC DETECT Data Oversight Committee (DOC) includes representatives from the NC DPH, UNC NC DETECT Team and NC Hospital Association.

\section{References}

1. Centers for Disease and Control Vital Signs, Morbidity and Mortality Weekly Report (MMWR) Oct. 2014.

2. Centers for Disease Control and Prevention. Web-based Injury Statistics Query and Reporting System (WISQARS) [Online]. (2003). National Center for Injury Prevention and Control, Centers for Disease Control and Prevention. www.cdc.gov/ncipc/wisqars.

3. Barell, Vita, et al. "An introduction to the Barell body region by nature of injury diagnosis matrix.” Injury Prevention 8.2 (2002): 91-96.

\section{*Jennifer L. Jones}

E-mail: jjones86@live.unc.edu 